state with baseline data, root cause analysis, and surveys to identify common and impactful failures. A Key Driver Diagram defined leading factors. Run charts and later SPC charts captured change over time. A communication board displays ongoing work; weekly huddles evaluate progress and barriers.

Results We met and exceeded goal since in 12/2017. We have sustained our improvement with an average of $92 \%$ as of 06/2019. This work also helped decrease time from referral to consult for all clinic referrals from 65.4 business to 21 business days. Additionally, this project helped our clinic lower no-show rates from $15 \%$ to $6 \%$ making it the lowest rate for the entire Specialty Center. Furthermore, it helped increase patient volume by an average of 30 more patients per month starting $07 / 17$ and $\$ 375,000$ increased revenue even before factoring in additional downstream revenue. During this period, patient satisfaction scores reached 93-100\%.

Conclusions Standardizing, empowering the care team, and improving communication across primary and specialty care brought relief to over 800 families, connecting suffering children with the right care at the right time.

\section{IMPLEMENTATION ANALYSIS OF A COMPREHENSIVE PATIENT SAFETY PROGRAM IN GUATEMALA}

${ }^{1}$ Henry Rice, ${ }^{2}$ Randall Lou-Meda, ${ }^{2}$ Sindy Mendez, ${ }^{1}$ Bria Hall, ${ }^{1}$ Isabelle Sico, ${ }^{1}$ Joy Noel Baumgartner. 'Duke Global Health Institute, USA; ${ }^{2}$ Fundanier, Roosevelt Hospital, Guatemala

\subsection{6/bmjoq-2019-ihi.6}

Background Although patient safety programs improve clinical outcomes in a variety of settings, little is known about factors affecting successful implementation of safety programs in low and middle-income countries (LMICs).

Objectives Our goal was to investigate the implementation of a comprehensive patient safety program for pediatric care in Guatemala.

Methods We performed a mixed-methods implementation analysis of a comprehensive patient safety program in 11 pediatric units at the Roosevelt Hospital in Guatemala. The safety program was contextualized to each unit, and included assessment of the safety culture, perioperative checklists, and targeted QI interventions. We performed qualitative analysis of implementation challenges using semistructured interviews with hospital staff $(n=82)$ using the Consolidated Framework for Implementation Research (CFIR), with follow-up focus group interviews one year later. We also measured staff views towards implementation challenges using the quantitative Evidence Based Practice Attitude Scale (EBPAS-36). All data were triangulated to identify emerging themes as well as barriers and facilitators to implementation.

Results We classified implementation challenges within 25 CFIR constructs. We identified several emerging themes:
1) High staff receptivity to safety programs, including recognition of relevance to their patient population; 2) Importance of increasing staff knowledge and awareness about patient safety, 3) Difficulties with high patient care demands; 4) Limited governance and leadership engagement; 5) Contextualization of safety programs to local needs.

Conclusions Our study provides insight into hospital staff perspectives on implementation challenges for patient safety programs in Guatemala. Definition of implementation challenges may enhance successful adoption of safety practices in lowresource settings.

\section{THE MULTIPLE SCLEROSIS CONTINUOUS QUALITY IMPROVEMENT COLLABORATIVE (MSCQI): INTERIM RESULTS OF THE 3-YEAR MULTI-CENTER PROSPECTIVE STEP-WEDGE RANDOMIZED RESEARCH STUDY}

Brant Oliver. Dartmouth Geisel School of Medicine, USA

10.1136/bmjoq-2019-ihi.7

Background MS-CQI is the first multi-center, randomized research study aiming to improve population health outcomes for people with multiple sclerosis (MS) using quality improvement (QI).

Objectives (1) benchmark performance and study variation in utilization and outcomes; (2) provide performance feedback to MS centers; and (3) compare effectiveness of QI versus usual care controls on improving outcomes.

Methods Four centers are participating following approximately 5,000 people with MS. We collect 21 Patient Reported Outcome Measures (PROMs) and 11 Electronic Health Record (EHR) measures. System-level de-identified EHR data is collected for all clinical encounters. Individual-level PROM data is collected from participants who consent. Centers are randomized to QI or usual care following a step-wedge randomized design beginning in Year 2.

Results MSCQI has recently completed Year 2. At the end of Year 1, EHR $n=2,755$ encounters, and PROM $n=269$ individuals (tables 1-3). There is significant variation in EHR findings, including proportion of patients on disease modifying therapy (DMT), MRI, ED, hospitalizations, urgent care, and relapses (table 2), as well as PROM findings (table 3) including depression, fatigue, cognition, sleep, communication, and work-related impairment. In Year 2, the first center (Center C) was randomized to QI. Center $\mathrm{C}$ has higher average depression and fatigue severity than the Collaborative, but has realized a reduction in quarterly relapse rate since beginning QI intervention in Quarter 4 (figure 1).

Conclusions MSCQI has succeeded in benchmarking systemlevel variation and has begun studying outcomes of QI intervention versus usual care. MSCQI has potential to improve 
Abstract 7 Table 1

\begin{tabular}{|c|c|c|c|c|c|}
\hline & $\begin{array}{l}\text { Center A (Rural } \\
\text { Community Hospital) }\end{array}$ & $\begin{array}{l}\text { Center B (Urban } \\
\text { Academic Center) }\end{array}$ & $\begin{array}{l}\text { Center C (Urban Large } \\
\text { Private Practice) }\end{array}$ & $\begin{array}{l}\text { Center D (Rural } \\
\text { Academic Center) }\end{array}$ & $\begin{array}{l}\text { MS-CQI } \\
\text { Collaborative (Total) }\end{array}$ \\
\hline Patients (n, \%) & $963(35.0)$ & $436(15.8)$ & $616(22.4)$ & $740(26.9)$ & $2,755(100)$ \\
\hline Age (mean, SD) & $52.2(12.0)$ & $49.2(13.3)$ & 49.7 (12.6) & $52.2(12.7)$ & $51.1(12.6)$ \\
\hline \multicolumn{6}{|l|}{$\operatorname{Sex}(n, \%)$} \\
\hline Male & $204(21.2)$ & $116(26.6)$ & $138(22.4)$ & $194(26.2)$ & $652(23.7)$ \\
\hline Female & $759(78.8)$ & $320(73.4)$ & $478(77.6)$ & $546(73.8)$ & $2,103(76.3)$ \\
\hline \multicolumn{6}{|l|}{ Comorbidities (n,\%) } \\
\hline Anxiety & $300(31.2)$ & 59 (13.5) & $110(17.9)$ & $144(19.5)$ & $613(22.3)$ \\
\hline Arthritis & $44(4.6)$ & $22(5.1)$ & $83(13.5)$ & $72(9.7)$ & $221(8.0)$ \\
\hline Asthma & $68(7.1)$ & $40(9.2)$ & $31(5.0)$ & $63(8.51)$ & $202(7.3)$ \\
\hline Back Pain & $139(14.4)$ & $24(5.5)$ & $56(9.1)$ & $110(14.9)$ & $329(11.9)$ \\
\hline COPD & $21(2.2)$ & $1(0.2)$ & $7(1.1)$ & $7(1.0)$ & $36(1.3)$ \\
\hline Cancer & $22(2.3)$ & $40(9.2)$ & $51(8.3)$ & 85 (11.5) & $198(7.2)$ \\
\hline Depression & $368(38)$ & $79(18.1)$ & $180(29.2)$ & $250 * 33.8)$ & $877(31.8)$ \\
\hline Diabetes & $69(7.2)$ & $28(6.4)$ & $46(7.5)$ & $53(7.2)$ & $196(7.1)$ \\
\hline Heart Disease & $26(2.7)$ & $24(5.5)$ & $30(4.9)$ & 86 (11.6) & $166(6.0)$ \\
\hline Hypercholesterolemia & $191(19.8)$ & $23(5.3)$ & $87(14.1)$ & $140(18.9)$ & $441(16.0)$ \\
\hline Hypertension & $228(23.7)$ & $106(24.3)$ & $193(31.3)$ & $172(23.2)$ & $699(25.4)$ \\
\hline Insomnia & $124(12.9)$ & $5(1.2)$ & $18(2.9)$ & $18(2.4)$ & $165(6.0)$ \\
\hline Irritable Bowel Syndrome & $36(3.7)$ & $16(3.7)$ & $6(1.0)$ & $27(3.7)$ & $85(3.1)$ \\
\hline Kidney Disease & $9(0.9)$ & $20(4.6)$ & $7(1.1)$ & $29(3.9)$ & $65(2.4)$ \\
\hline Liver Disease & $2(0.2)$ & $0(0)$ & $4(0.7)$ & $16(2.2)$ & $22(0.8)$ \\
\hline Lung Disease & $2(0.2)$ & $2(0.5)$ & $11(1.8)$ & $16(2.2)$ & $31(1.1)$ \\
\hline Migraine & $203(21.1)$ & $36(8.3)$ & $186(30.2)$ & $150(20.3)$ & $575(20.9)$ \\
\hline Osteoporosis & $43(4.5)$ & $37(8.5)$ & $7(1.1)$ & $31(4.2)$ & $118(4.3)$ \\
\hline Stroke (CVA) & $14(1.5)$ & $1(0.2)$ & $20(3.3)$ & $12(1.62)$ & $47(1.7)$ \\
\hline Thyroid Disease & $85(8.8)$ & $64(14.7)$ & $86(14.0)$ & 85 (11.5) & $320(11.6)$ \\
\hline Ulcer & $1(0.1)$ & $1(0.2)$ & $6(1.0)$ & $4(0.5)$ & $12(0.4)$ \\
\hline Other & $27(2.8)$ & $10(2.3)$ & $3(0.5)$ & $1(0.1)$ & $41(1.5)$ \\
\hline
\end{tabular}

\section{Abstract 7 Table 2}

\begin{tabular}{|c|c|c|c|c|c|c|c|}
\hline Proportion on DMT & $\begin{array}{l}\text { Center A (Rural } \\
\text { Community Hospital) }\end{array}$ & $\begin{array}{l}\text { Center B (Urban } \\
\text { Academic Center) }\end{array}$ & $\begin{array}{l}\text { Center C (Urban } \\
\text { Private Practice) }\end{array}$ & $\begin{array}{l}\text { Center D (Rural } \\
\text { Academic Center) }\end{array}$ & MS-CQI (Total) & ANOVA $p$ & Significant \\
\hline Patients $(n, \%)$ & $963(35.0)$ & $436(15.8)$ & $616(22.4)$ & $740(26.9)$ & $2,755(100)$ & & \\
\hline Proportion of patients $(n, \%)$ & $742(77.1)$ & $357(81.9)$ & $520(84.4)$ & $464(62.7)$ & $2,083(75.6)$ & $<0.0001$ & * \\
\hline Proportion with at least $1 \mathrm{MRI}$ & Center A & Center B & Center C & Center D & MS-CQI (Total) & $\mathrm{p}$ & Significant \\
\hline Patients $(n, \%)$ & $963(35.0)$ & $436(15.8)$ & $616(22.4)$ & $740(26.9)$ & $2,755(100)$ & & \\
\hline Brain MRI (n, \%) & $623(64.7)$ & $315(72.3)$ & $375(60.9)$ & $387(52.3)$ & $1,700(61.7)$ & $<0.0001$ & * \\
\hline C-Spine MRI $(n, \%)$ & $371(38.5)$ & $117(26.8)$ & $189(30.7)$ & $156(21.1)$ & $833(30.2)$ & $<0.0001$ & * \\
\hline T-Spine MRI $(n, \%)$ & $158(16.4)$ & $93(21.3)$ & $136(22.1)$ & $68(9.2)$ & $455(16.5)$ & $<0.0001$ & * \\
\hline \# of Hospitalizations & Center A & Center B & Center C & Center D & MS-CQI (Total) & $\mathrm{p}$ & Significant \\
\hline Patients (n, \%) & $963(35.0)$ & $436(15.8)$ & $616(22.4)$ & $740(26.9)$ & $2,755(100)$ & & \\
\hline $0(n, \%)$ & $916(95.1)$ & 408 (93.6) & $557(90.4)$ & $630(85.1)$ & $2,511(91.1)$ & $<0.0001$ & * \\
\hline $1(n, \%)$ & $32(3.3)$ & $25(5.7)$ & $52(8.4)$ & $81(11.0)$ & $190(6.9)$ & & \\
\hline $2+(n, \%)$ & $15(1.6)$ & $3(0.7)$ & 7 (1.1) & $2(3.9)$ & $54(2.0)$ & & \\
\hline$\#$ of ED visits & Center A & Center B & Center C & Center D & MS-CQI (Total) & $\mathrm{p}$ & Significant \\
\hline Patients (n,\%) & $963(35.0)$ & $436(15.8)$ & $616(22.4)$ & $740(26.9)$ & $2,755(100)$ & & \\
\hline $0(n, \%)$ & $869(90.2)$ & $386(88.5)$ & $587(95.3)$ & $649(87.7)$ & 2,491 (90.4) & $<0.0001$ & * \\
\hline $1(n, \%)$ & $52(5.4)$ & $41(9.4)$ & $26(4.2)$ & $67(9.1)$ & $186(6.8)$ & & \\
\hline $2+(n, \%)$ & $42(4.4)$ & $9(2.1)$ & $3(0.5)$ & $24(3.2)$ & $78(2.8)$ & & \\
\hline \# of Urgent Care visits & Center A & Center B & Center C & Center D & MS-CQI (Total) & $\mathrm{p}$ & Significant \\
\hline Patients (n,\%) & $963(35.0)$ & $436(15.8)$ & $616(22.4)$ & $740(26.9)$ & $2,755(100)$ & & \\
\hline
\end{tabular}


Abstracts

\begin{tabular}{|c|c|c|c|c|c|c|c|}
\hline $0(n, \%)$ & 940 (97.6) & 421 (96.6) & 609 (98.86) & 705 (95.3) & 2,675 (97.1) & $<0.0001$ & * \\
\hline $1(n, \%)$ & $18(1.9)$ & $9(2.1)$ & $6(1.0)$ & $26(3.5)$ & $59(2.1)$ & & \\
\hline $2+(n, \%)$ & $5(0.5)$ & $6(1.4)$ & $1(0.2)$ & $9(1.2)$ & $21(0.8)$ & & \\
\hline Outcomes (Means) & Center A & Center B & Center C & Center D & MS-CQI (Total) & $\mathrm{p}$ & Significant \\
\hline Patients $(n, \%)$ & $963(35.0)$ & $436(15.8)$ & $616(22.4)$ & $740(26.9)$ & $2,755(100)$ & & \\
\hline Hospitalized (mean,SD) & $0.07(0.40)$ & $0.07(0.28)$ & $0.11(0.39)$ & $0.21(0.63)$ & $0.12(0.46)$ & $<0.0001$ & * \\
\hline ED Visit (mean,SD) & $0.19(0.74)$ & $0.14(0.45)$ & $0.05(0.24)$ & $0.21(0.78)$ & $0.16(0.63)$ & $<0.0001$ & * \\
\hline UC Visit (mean,SD) & $0.03(0.21)$ & $0.05(0.27)$ & $0.01(0.13)$ & $0.06(0.33)$ & $0.04(0.25)$ & 0.0007 & * \\
\hline \# of Relapses (Exacerbations) & Center A & Center B & Center C & Center D & MS-CQI (Total) & $\mathrm{p}$ & Significant \\
\hline Patients $(n, \%)$ & $963(35.0)$ & $436(15.8)$ & $616(22.4)$ & $740(26.9)$ & $2,755(100)$ & & \\
\hline $0(n, \%)$ & 892 (92.6) & $385(88.3)$ & $512(83.1)$ & $703(95.0)$ & $2,492(90.5)$ & $<0.0001$ & * \\
\hline $1(n, \%)$ & $62(6.4)$ & $49(11.2)$ & $97(15.8)$ & $34(4.6)$ & $242(8.8)$ & & \\
\hline $2+(n, \%)$ & $9(0.9)$ & $2(0.5)$ & $7(1.1)$ & $3(0.4)$ & $21(0.8)$ & & \\
\hline Relapses (Annualized) & Center A & Center B & Center C & Center D & MS-CQI (Total) & $\mathrm{p}$ & Significant \\
\hline Patients $(n, \%)$ & $963(35.0)$ & $436(15.8)$ & $616(22.4)$ & $740(26.9)$ & $2,755(100)$ & & \\
\hline Relapse Rate (mean,SD) & $0.08(0.32)$ & $0.12(0.34)$ & $0.18(0.43)$ & $0.05(0.24)$ & $0.10(0.34)$ & $<0.0001$ & * \\
\hline
\end{tabular}

Key: DMT = Disease modifying therapy

MRI = Magnetic resonance imaging

C-Spine $=$ Cervical spinal cord

$\mathrm{T}$-Spine $=$ Thoracic spinal cord

$\mathrm{ED}=$ Emergency Department

$U C=$ Urgent care visit

ANOVA = Analysis of Variance

Abstract 7 Table 3

\begin{tabular}{|c|c|c|c|c|c|c|c|c|c|c|c|c|c|c|c|c|}
\hline \multirow[t]{3}{*}{ Survey Name } & \multicolumn{3}{|c|}{$\begin{array}{c}\text { Center A (Rural } \\
\text { Community Hospital) }\end{array}$} & \multicolumn{3}{|c|}{$\begin{array}{l}\text { Center B (Urban } \\
\text { Academic Center) }\end{array}$} & \multicolumn{3}{|c|}{$\begin{array}{l}\text { Center C (Urban } \\
\text { Private Practice) }\end{array}$} & \multicolumn{3}{|c|}{ Center D } & \multicolumn{3}{|c|}{$\begin{array}{l}\text { MS-CQI } \\
\text { (Total) }\end{array}$} & \multirow[b]{3}{*}{$\begin{array}{l}\text { ANOVA } \\
\text { (p) }\end{array}$} \\
\hline & \multicolumn{3}{|c|}{$\begin{array}{l}\text { Rural Community } \\
\text { Hospital }\end{array}$} & \multicolumn{3}{|c|}{$\begin{array}{c}\text { Urban Academic } \\
\text { Center }\end{array}$} & \multicolumn{3}{|c|}{ Urban Private Practice } & \multicolumn{3}{|c|}{$\begin{array}{l}\text { Rural Academic } \\
\text { Center }\end{array}$} & & & & \\
\hline & $\mathrm{N}$ & Mean & SD & $\mathrm{N}$ & Mean & SD & $\mathrm{N}$ & Mean & SD & N & Mean & SD & $\mathrm{N}$ & Mean & SD & \\
\hline $\begin{array}{l}\text { Patient Determined Disease Steps (PDDS)- } \\
\text { Most Recent }\end{array}$ & 57 & 1.5 & 1.8 & 71 & 1.5 & 2.2 & 57 & 1.6 & 1.8 & 45 & 2.3 & 2.3 & 230 & 1.7 & 2.0 & \\
\hline Depression Severity (PHQ-9) & 47 & 6.0 & 3.6 & 73 & 5.8 & 5.1 & 66 & 5.7 & 4.3 & 58 & 8.2 & 5.2 & 244 & 6.4 & 4.8 & * \\
\hline $\begin{array}{l}\text { Fatigue Severity (PROMIS Fatigue-MS Raw } \\
\text { Score) }\end{array}$ & 44 & 21.4 & 5.5 & 72 & 19.9 & 7.2 & 65 & 21.2 & 7.3 & 53 & 24.4 & 8.1 & 234 & 21.6 & 7.3 & * \\
\hline Neuro-Qol Anxiety & 66 & 17.2 & 6.6 & 84 & 17.2 & 6.2 & 66 & 16.0 & 5.7 & 55 & 19.2 & 7.5 & 271 & 17.4 & 6.5 & 0.060 \\
\hline Neuro-Qol Cognitive & 65 & 29.6 & 7.6 & 83 & 31.9 & 7.1 & 66 & 32.2 & 6.2 & 55 & 28.9 & 7.8 & 269 & 30.8 & 7.3 & * \\
\hline Neuro-Qol Lower Exetremity Function & 59 & 35.1 & 6.0 & 70 & 34.8 & 7.7 & 57 & 34.9 & 7.3 & 46 & 33.2 & 7.8 & 232 & 34.6 & 7.2 & \\
\hline Neuro-Qol Upper Extremity Function & 59 & 37.7 & 4.1 & 69 & 38.3 & 3.4 & 57 & 37.6 & 4.9 & 46 & 37.4 & 3.4 & 231 & 37.8 & 4.0 & \\
\hline Neuro-Qol Stigma & 56 & 11.5 & 4.2 & 68 & 11.9 & 5.2 & 59 & 12.4 & 5.3 & 44 & 14.1 & 5.9 & 227 & 12.4 & 5.2 & \\
\hline $\begin{array}{l}\text { Neuro-Qol Social Roles and Activities - } \\
\text { Participation }\end{array}$ & 55 & 32.3 & 6.4 & 67 & 32.6 & 7.3 & 59 & 31.7 & 7.2 & 44 & 29.8 & 7.6 & 225 & 31.8 & 7.2 & \\
\hline $\begin{array}{l}\text { Neuro-Qol Social Roles and Activities - } \\
\text { Satisfaction }\end{array}$ & 53 & 30.3 & 7.1 & 67 & 29.5 & 8.2 & 59 & 28.2 & 8.2 & 44 & 26.4 & 8.1 & 223 & 28.8 & 8.0 & \\
\hline Neuro-Qol Sleep & 50 & 18.4 & 5.4 & 64 & 18.8 & 6.4 & 58 & 19.1 & 5.6 & 45 & 21.5 & 5.6 & 217 & 19.3 & 5.9 & * \\
\hline Neuro-Qol Communication & 50 & 88.0 & 14.1 & 64 & 93.4 & 11.7 & 57 & 89.3 & 10.5 & 45 & 88.6 & 11.8 & 216 & 90.1 & 12.1 & * \\
\hline Treatment Satisfaction (TSQM-9) Effectiveness & 37 & 70.6 & 20.5 & 31 & 69.5 & 19.4 & 27 & 80.0 & 13.4 & 23 & 74.0 & 20.2 & 118 & 73.1 & 18.9 & \\
\hline Treatment Satisfaction (TSQM-9) Convenience & 37 & 74.0 & 21.7 & 31 & 72.7 & 19.6 & 27 & 77.9 & 16.2 & 23 & 81.5 & 16.6 & 118 & 76.0 & 19.1 & \\
\hline Treatment Satisfaction (TSQM-9) Global & 37 & 69.9 & 22.5 & 31 & 69.5 & 20.2 & 27 & 81.6 & 13.9 & 23 & 75.8 & 20.0 & 118 & 73.6 & 20.1 & \\
\hline WWPAI Impairment While Working & 24 & 10.8 & 12.5 & 25 & 13.6 & 19.1 & 27 & 28.8 & 30.3 & 15 & 29.3 & 23.8 & 91 & 20.0 & 23.6 & * \\
\hline WWPAI Activity Impairment & 25 & 18.0 & 22.0 & 25 & 23.2 & 22.7 & 28 & 29.6 & 28.0 & 16 & 26.6 & 21.2 & 94 & 24.3 & 24.0 & \\
\hline $\begin{array}{l}\text { My MS Relapse- Did you experience a } \\
\text { relapse recently? }\end{array}$ & 48 & 0.5 & 0.8 & 58.0 & 0.6 & 1.2 & 53.0 & 0.3 & 0.7 & 46.0 & 0.8 & 1.4 & 205.0 & 0.5 & 1.1 & \\
\hline
\end{tabular}

${ }^{*} p \leq 0.05$ 


\begin{tabular}{|c|c|c|}
\hline & $\begin{array}{c}\text { Center A } \\
\text { (Rural Community Hospital) }\end{array}$ & $\begin{array}{c}\text { Center B } \\
\text { (Urban Academic Center) }\end{array}$ \\
\hline Patients (n,\%) & $963(35.0)$ & $436(15.8)$ \\
\hline Age (mean, SD) & $52.2(12.0)$ & $49.2(13.3)$ \\
\hline \multicolumn{3}{|l|}{$\operatorname{Sex}(n, \%)$} \\
\hline Male & $204(21.2)$ & $116(26.6)$ \\
\hline Female & $759(78.8)$ & $320(73.4)$ \\
\hline \multicolumn{3}{|l|}{ Comorbidities $(\mathrm{n}, \%)$} \\
\hline Anxiety & $300(31.2)$ & $59(13.5)$ \\
\hline Arthritis & $44(4.6)$ & $22(5.1)$ \\
\hline Asthma & $68(7.1)$ & $40(9.2)$ \\
\hline Back Pain & $139(14.4)$ & $24(5.5)$ \\
\hline COPD & $21(2.2)$ & $1(0.2)$ \\
\hline Cancer & $22(2.3)$ & $40(9.2)$ \\
\hline Depression & $368(38)$ & $79(18.1)$ \\
\hline Diabetes & $69(7.2)$ & $28(6.4)$ \\
\hline Heart Disease & $26(2.7)$ & $24(5.5)$ \\
\hline Hypercholesterolemia & $191(19.8)$ & $23(5.3)$ \\
\hline Hypertension & $228(23.7)$ & $106(24.3)$ \\
\hline Insomnia & $124(12.9)$ & $5(1.2)$ \\
\hline Irritable Bowel Syndrome & $36(3.7)$ & $16(3.7)$ \\
\hline Kidney Disease & $9(0.9)$ & $20(4.6)$ \\
\hline Liver Disease & $2(0.2)$ & $0(0)$ \\
\hline Lung Disease & $2(0.2)$ & $2(0.5)$ \\
\hline Migraine & $203(21.1)$ & $36(8.3)$ \\
\hline Osteoporosis & $43(4.5)$ & $37(8.5)$ \\
\hline Stroke (CVA) & $14(1.5)$ & $1(0.2)$ \\
\hline Thyroid Disease & $85(8.8)$ & 64 (14.7) \\
\hline Ulcer & $1(0.1)$ & $1(0.2)$ \\
\hline Other & $27(2.8)$ & $10(2.3)$ \\
\hline
\end{tabular}

Abstract 7 Figure 1 Year 2 Center C Results: Statistical Process Control (SPC) analyses describing the variation in performance compared to the overall average performance of the MSCQI Collaborative. Blue squares represent point esitimates, the green line represents the overall average, and red lines denote 3 Sigma upper and lower control limits. Points outside control limits (shown in red) indicate non-random (special cause) variation

MS population health outcomes using improvement science methods.

\section{TRANSITIONAL CARE CENTER: A CENTER FOR MEDICAL AND NON-MEDICAL EXCELLENCE}

${ }^{1}$ Nkem Chukwumerije, ${ }^{2}$ Parnika Kodali. 'SCAL Permanente Medical Group, USA; ${ }^{2}$ Kaiser Hospital Health Plan, USA

\subsection{6/bmjoq-2019-ihi.8}

Background In 2016, in PCMC, only 75\% high risk patients were receiving post hospital follow up care. Given that $24 \%$ of our high-risk patients are readmitted, it is fair to assume that no follow up results in higher utilization. For those that did receive follow up, it was variable depending on the physician. Primary care offices did not have a robust process for social needs evaluation. Hence, patients used more ED services for social needs. Programs that address both medical and non medical needs of patients show higher success but are often limited.

Objectives Reduce utilization by addressing medical and nonmedical needs of high-risk patients.

Methods TCC appointment consists of: 40-minute medical assessment by a physician, pharmacist lead medication reconciliation, social worker assessment for social and behavioral needs, care navigator to secure resources, and care coordinator to manage the care plan for upto 90 days.

Results A single centered retrospective study was used to evaluate pre and post utilization of high risk patients that were treated in TCC and a similar control group that received care with a PCP. The demographics of both groups were evaluated to ensure similar representation. The results showed a significant decrease in utilization for the TCC patients compared to 